\title{
Rigidity analysis of kenaf thermoplastic composites using Halpin-Tsai equation
}

\begin{abstract}
In this paper, the stiffness mechanical property of natural fiber reinforced thermoplastic composites is analyzed using composite micromechanical model. Kenaf natural fiber is selected as the reinforcement material in the composites construction while three types of commonly used automotive grade thermoplastic matrices, namely polypropylene, acrylonitrile butadiene styrene and polyamide 6 were selected to be reinforced with kenaf fibers. Their stiffness property was later analyzed using Halpin-Tsai micromechanical model at varying fiber content and fiber aspect ratio conditions. In all cases, theoretical results show that the kenaf reinforced thermoplastic composites stiffness increased linearly as the fiber contents were increased. Apart from that, results also show that the stiffness property also increases as the fiber aspect ratio was increased. Higher final composites stiffness property was also observed as stiffness matrix material is utilized in the composites formulation. The prediction results also provided valuable and quick insight as well as cost effective alternative to composite designers in assessing the stiffness performance of natural fiber composites especially those which are reinforced with thermoplastic matrices compared to conventional experimental technique for automotive product development purposes in addition to identifying the optimal parameter to be put into focus in their composites design to achieve the intended design performance specifications.
\end{abstract}

Keyword: Halpin-Tsai equation; Kenaf thermoplastic composites; Rigidity; Rigidity analysis 